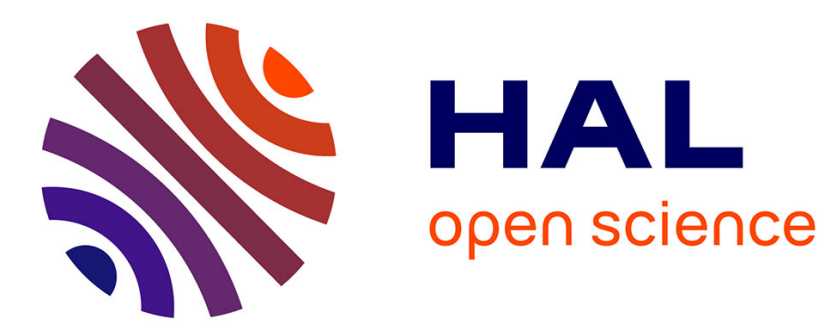

\title{
Le rôle du figement dans le traitement de la synonymie au sein du champ de la colère
}

Julie Sorba, Vannina Goossens

\section{To cite this version:}

Julie Sorba, Vannina Goossens. Le rôle du figement dans le traitement de la synonymie au sein du champ de la colère. Lingvisticae Investigationes, 2016, 39 (1), pp.1 - 26. 10.1075/li.39.1.01sor . hal-01844290

\author{
HAL Id: hal-01844290 \\ https://hal.science/hal-01844290
}

Submitted on 24 Apr 2020

HAL is a multi-disciplinary open access archive for the deposit and dissemination of scientific research documents, whether they are published or not. The documents may come from teaching and research institutions in France or abroad, or from public or private research centers.
L'archive ouverte pluridisciplinaire HAL, est destinée au dépôt et à la diffusion de documents scientifiques de niveau recherche, publiés ou non, émanant des établissements d'enseignement et de recherche français ou étrangers, des laboratoires publics ou privés. 


\title{
Le rôle du figement dans le traitement de la synonymie au sein du champ de la colère
}

\author{
Julie Sorba ${ }^{1}$ et Vannina Goossens ${ }^{2}$ \\ ${ }^{1}$ Univ. Grenoble Alpes - Lidilem, ${ }^{2}$ ENS de Lyon - ICAR
}

\section{Introduction $^{\mathrm{i}}$}

Notre travail a pour objectif de mettre en évidence les caractéristiques spécifiques des lexies d'affect synonymes à travers l'étude de leur figement. Nous nous intéressons ici à quatre lexies françaises relevant du champ de la colère : les noms rage et fureur ainsi que les adjectifs dérivés rageur et furieux. Comme la notion de figement regroupe un ensemble de phénomènes aux caractéristiques variées, nous privilégions dans ce cadre un type de figement particulier: les collocations. Nous les définissons, selon la tradition continentale, comme des «associations lexicales privilégiées et sémantiquement compositionnelles » (Tutin, 2013, 47).

L'étude de la combinatoire de ces quatre lexies est menée sur un vaste corpus, ce qui nous permet d'opérer à trois niveaux (sémantique, syntaxique et textuel) et d'élargir de manière systématique nos analyses à la dimension discursive. En effet, si l'intérêt de cette démarche d'analyse combinatoire n'est plus à prouver au niveau phrastique pour l'étude des noms d'affect ${ }^{\mathrm{ii}}$, son extension au niveau textuel n'a pas encore été pleinement exploitée. On pourrait penser que plus l'on s'éloigne d'un mot pivot, plus la liberté de choix de ses cooccurrents est grande. Or, il s'avère que certains mots ou contenus sont plus probables que d'autres dans l'environnement textuel de la lexie étudiée $e^{\text {iii }}$. À la prévisibilité du contexte immédiat du mot pivot s'ajoute ainsi celle d'autres éléments dans le paragraphe voire le texte (cf. Blumenthal, 2002 ; Novakova \& Sorba, 2013a et 2013b).

Après avoir présenté notre cadre théorique et méthodologique ainsi que le corpus retenu pour l'étude, nous examinerons les profils lexicaux, syntaxiques et textuels des quatre lexies étudiées afin de mettre en évidence les comportements distincts de ces lexies présentées habituellement comme synonymes. L'ensemble de ces données est regroupé dans un profil discursif qui spécifie le comportement de la lexie au sein des 
textes. Nous faisons ainsi l'hypothèse que le profil discursif de chacune des lexies fournit des éléments pertinents pour distinguer les synonymes entre eux.

\section{Cadre théorique, méthodologie, corpus}

\subsection{Cadre théorique}

La présente étude s'inscrit dans une perspective fonctionnelle qui analyse la manière dont les niveaux linguistiques (syntaxique, sémantique, textuel) interagissent au service de la structuration et de la hiérarchisation de l'information. De ce fait, elle intègre de manière systématique la dimension discursive à l'analyse du comportement syntaxique et sémantique des lexies examinées (cf. Van Valin \& LaPolla, $1997^{\mathrm{iv}}$ ). Nous souhaitons ainsi élargir la perspective des précédentes études nombreuses sur les lexies d'émotion qui partent de la syntaxe pour étudier le sens (comme Gross, 1995) ou, inversement, d'ensemble sémantiquement homogènes de lexies pour examiner leurs propriétés syntaxiques (comme Yannick Mathieu, 2000).

Nous avons sélectionné deux noms d'affect ${ }^{\mathrm{v}}$, fureur et rage, désignant tous deux des affects interpersonnels causés, réactifs, ponctuels et de forte intensitévi À la différence de la colère, la rage et la fureur ont fait l'objet de moins d'études systématiques ${ }^{\mathrm{vii}}$.

Les outils lexicographiques les présentent généralement comme synonymes. Ainsi, dans l'article consacré à rage, fureur est le seul synonyme proposé par le Petit Robert (Rey-Debove \& Rey (Eds.), 2014). On le retrouve aux côtés de colère et furie dans le Trésor de la Langue Française informatisé (TLFi, Imbs \& Quémada (Eds.), 1994), présentés comme « synonymes partiels ». Pour fureur, on ne trouve aucune mention de synonymes dans le TLFI pour l'acception d'affect; dans le Petit Robert (PR), les collocations entrer, être, mettre en fureur sont rapprochées d'enrager. En ce qui concerne les définitions lexicographiques, celles du TLFi, plus détaillées que celles du $\mathrm{PR}^{\text {viii }}$, incluent l'affect au sein d'un scénario affectif plus ou moins structuré (l'expression d'une cause ou d'une conséquence de l'affect y apparait) :

- rage : «État affectif pouvant se manifester par une grande violence verbale ou physique, ou par un silence hargneux fait de colère, de ressentiment ou de haine, qui est généralement causé par un sentiment d'impuissance devant une situation frustrante » (expression de la cause et de la conséquence) ;

- fureur: "Colère intense, aux effets souvent démesurés » (expression de la conséquence seule). 
Nous avons également choisi d'inclure dans nos analyses l'étude des deux adjectifs dérivés furieux et rageur, la combinatoire des adjectifs étant nettement moins étudiée que celle des noms et des verbes à ce jour. Dans les outils lexicographiques, la situation est sensiblement différente puisqu'ils ne sont pas présentés comme synonymes potentiels. Pour rageur, ce sont les lexies coléreux et irascible (PR) qui sont proposés. Pour furieux, la liste est nettement plus fournie dans le PR avec furibond, fumasse, furax et furibard alors que le TLFi n'en propose aucun, comme pour rageur. Les définitions pour autant sont particulièrement proches :

- rageur : "sujet à des accès de colère, de hargne » $(\mathrm{PR})$, " qui est en rage » (TLFi) lorsque le premier actant est animé ; « qui dénote la colère, la mauvaise humeur » (PR), « qui exprime la mauvaise humeur » (TLFi) lorsque le premier actant est une métonymie (partie du corps - oeil rageur -, action - écriture rageuse -, etc.).

- furieux: «En proie à une folle colère » pour un premier actant animé, «qui dénote une folle colère » pour un premier actant métonymique (PR); « qui est en proie à une très grande colère, qui exprime la fureur $»$ (TFLi).

La démarche combinatoire adoptée ici permettra de discriminer ces lexies les unes des autres afin de compléter les observations délivrées par les outils lexicographiques.

\subsection{Méthodologie}

L'objectif de notre travail est de mettre en évidence les propriétés spécifiques de ces lexies sémantiquement proches qui décrivent un état intense ayant pour conséquence des comportements violents. Lors d'études antérieures portant sur les noms, les lexies rage et fureur n'avaient pu être distinguées. En effet, ces deux lexies appartiennent à la classe des " noms d'affect causés et dirigés par un objet » et dans la sous-catégorie des « noms qui admettent la possession et qui sont « voulus » ou en tous cas " assumés par l'expérienceur » (classe C. b., Goossens, 2005). De plus, elles n'acceptent pas de véhiculer d'autres acceptions régulières au sein de la classe des noms d'affect que l'interprétation d'affect (Goossens, 2011).

L'objectif est donc ici de mettre en évidence les caractéristiques de lexies sémantiquement proches en analysant, comme dans ces études, la combinatoire syntaxique et lexicale mais en élargissant le champ d'analyse au niveau textuel. Nous cherchons ainsi à établir, pour chacune des lexies étudiées, aux niveaux phrastique ou transphrastique selon les cas :

- le profil lexical, qui réunit les associations sémantiques privilégiées ;

- le profil syntaxique, qui analyse les structures actancielles des lexies en situation de figement; 
- le profil textuel, qui étudie l'impact des genres textuels sur le figement ainsi que les positions privilégiées par les lexies dans le texte.

Cette étude nous permet d'élaborer le profil discursif de chacune des lexies, pour lequel nous émettons l'hypothèse qu'il présente une spécificité propre à chacune d'entre elles.

\subsection{Corpus d'étude et approche statistique}

Notre corpus d'étude en langue française contemporaine inclut un ensemble de textes littéraires postérieurs à 1950 (environ 16 millions de mots) et deux années de parution des quotidiens Le Monde, Le Figaro, Libération, Ouest-France (environ 100 millions de mots, période 2007-2008). Ce corpus, annoté syntaxiquement, a été construit dans le cadre du projet ANR-DFG EMOLEX ${ }^{\mathrm{ix}}$. L'interface d'interrogation EmoConc permet d'extraire les lexicogrammes de chacune des lexies, c'est-à-dire la sélection de leurs cooccurrents spécifiques à partir du degré d'affinité combinatoire existant entre deux mots dans un corpus. Ce degré d'affinité est appréhendé sur la base du calcul de $\log _{\text {-likelihood }}$. La figure 1 ci-dessous représente le lexicogramme ainsi obtenu pour la lexie rageur.

\begin{tabular}{|c|c|c|c|c|c|c|}
\hline 11 & 12 & $f$ & f1 & f2 & am.log.likelihood & r.log.likelihood \\
\hline rageur_.* & poing_N & 30 & 790 & 11770 & 352,1569 & 1 \\
\hline rageur_:" & geste_N & 25 & 790 & 50976 & 211,0606 & 2 \\
\hline rageur_: & riff_N & 10 & 790 & 686 & 152,1087 & 3 \\
\hline rageur_.* & smash_N & 8 & 790 & 252 & 134,2574 & 4 \\
\hline rageur_: & coup_N & 18 & 790 & 257035 & 82,8924 & 5 \\
\hline rageur_. & ton_N & 9 & 790 & 33968 & 64,7976 & 6 \\
\hline rageur_:* & talon_N & 6 & 790 & 6484 & 58,1004 & 7 \\
\hline rageur_.* & souffle_N & 6 & 790 & 16486 & 46,9527 & 8 \\
\hline rageur_: & vent_N & 7 & 790 & 42575 & 43,7863 & 9 \\
\hline rageur_.' & pied_N & 8 & 790 & 98903 & 38,9705 & 10 \\
\hline rageur_: & livre_N & 6 & 790 & 158196 & 20,5764 & 11 \\
\hline rageur_." & plus_ADV & 10 & 790 & 790861 & 15,1911 & 12 \\
\hline 11 & 12 & $f$ & $\mathrm{f1}$ & 12 & am.log,llikelihood & r.log.likelihood \\
\hline
\end{tabular}

Figure 1 : Lexicogramme de la lexie rageur (Emoconc)

Le profil combinatoire de la lexie, qui réunit son voisinage sémanticosyntaxique spécifique (Blumenthal, 2002, 2007), est par la suite élaboré en deux temps. La première étape de sélection est automatique, puisque ne sont extraits du corpus que les cooccurrents spécifiques sur la base du calcul de log-likelihood. La seconde étape 
est manuelle. En effet, seuls les cooccurrents des lexies utilisées dans une interprétation d'affect sont conservés : les occurrences où les lexies ne désignent par un affect, comme dans vent rageur par exemple, sont ainsi écartées de notre corpus d'étude. Il en est de même pour les cooccurrences ne formant pas une collocation, selon la définition donnée en introduction. Ainsi, par exemple, les cooccurrences entre un nom d'affect et un mot grammatical sont écartées ${ }^{\mathrm{xi}}$. Après ce nettoyage nous obtenons, pour les quatre lexies de notre étude, un total de 44 collocatifs et de 367 séquences textuelles exploitables présentées ci-dessous (tableau 1).

\begin{tabular}{|c|c|c|}
\hline Pivot & Collocatifs & Séquences \\
\hline Rage & 16 & 134 \\
\hline Fureur & 11 & 88 \\
\hline Rageur & 7 & 44 \\
\hline Furieux & 10 & 101 \\
\hline Totaux & 44 & 367 \\
\hline
\end{tabular}

Tableau 1 : Nombre de collocatifs et de séquences textuelles étudiées

À première vue, on constate une inversion des fréquences respectives entre noms et adjectifs. En effet, rage est plus fréquent que fureur mais, pour les adjectifs, c'est furieux qui est plus fréquent que rageur. D'autre part, on observe, en ce qui concerne la productivité, une grande disparité entre le nom et l'adjectif pour le couple rage/rageur (16 collocatifs pour 134 occ. vs 7 collocatifs pour 44 occ.), alors que le couple fureur/furieux est très proche au niveau de la fréquence (11 collocatifs pour 88 occ. vs 10 collocatifs pour $101 \mathrm{occ})$.

\section{Profil lexical}

Le profil lexical de chacune des lexies s'élabore par l'analyse des dimensions sémantiques véhiculées par leurs collocatifs respectifs. Nous verrons dans un premier temps quelles sont les associations sémantiques partagées par les couples de synonymes ragelfureur et rageur/furieux et celles qui apparaissent comme spécifiques à chacune de ces lexies dans leurs collocations. Ensuite, nous examinerons les particularités sémantiques qui se dégagent de l'étude des collocatifs.

\subsection{Grille d'analyse sémantique}


À partir des travaux de Goossens, 2005 et de Tutin et al., 2006 a été élaborée une grille d'analyse sémantique des collocations des noms, verbes et adjectifs d'affect. Cette grille réunit un ensemble de huit dimensions sémantiques et de leurs valeurs qui peuvent être véhiculées par les collocatifs des lexies d'affect. Nous illustrons quelques dimensions sémantiques dans le tableau 2 ci-dessous.

\begin{tabular}{|l|l|l|}
\hline \multicolumn{1}{|c|}{ Dimensions } & \multicolumn{1}{c|}{ Valeurs } & \multicolumn{1}{c|}{ Exemples } \\
\hline Intensité & Fort $^{\text {xii }}$ & Fureur noire \\
\hline \multirow{2}{*}{ Contrôle } & Émotion & Ravaler sa rage \\
\cline { 2 - 3 } & Manifestation & Libérer sa rage \\
\hline \multirow{2}{*}{ Causativité } & Neutre & Rendre furieux \\
\cline { 2 - 3 } & Phasique & Susciter la fureur \\
\hline \multirow{2}{*}{ Manifestation } & Physique & Coup de poing rageur \\
\cline { 2 - 3 } & Verbale & Ton furieux \\
\hline
\end{tabular}

Tableau 2 : Grille d'analyse des dimensions et valeurs sémantiques des collocatifs

\subsection{Associations sémantiques : dimensions sémantiques spécifiques}

\subsubsection{Les noms rage et fureur}

Les graphiques des figures 2 et 3 présentent la répartition au sein des profils combinatoires $^{\text {xiii }}$ des dimensions sémantiques véhiculées par les collocatifs spécifiques des lexies rage et fureur. 


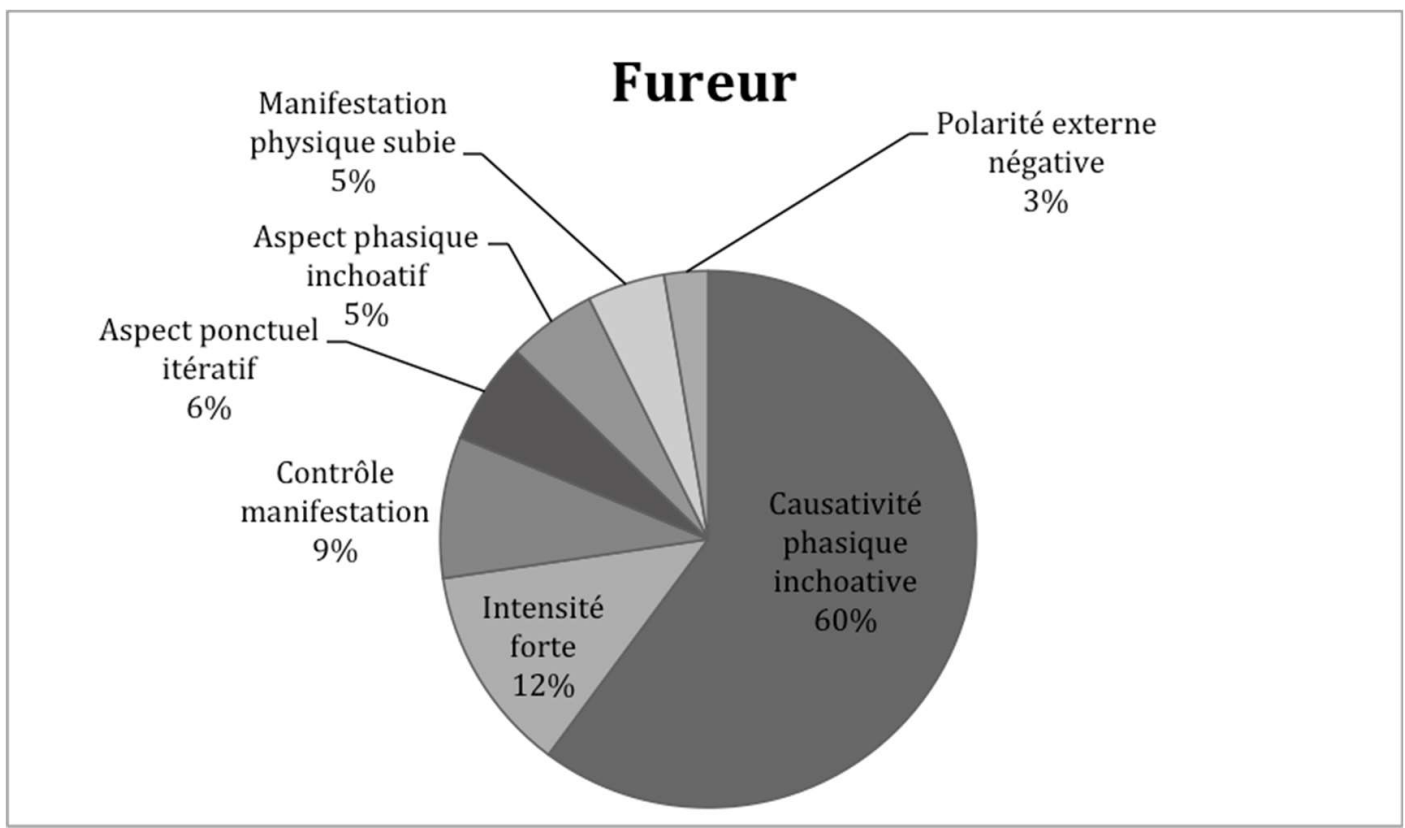

Figure 2 : Dimensions sémantiques des collocatifs spécifiques de fureur

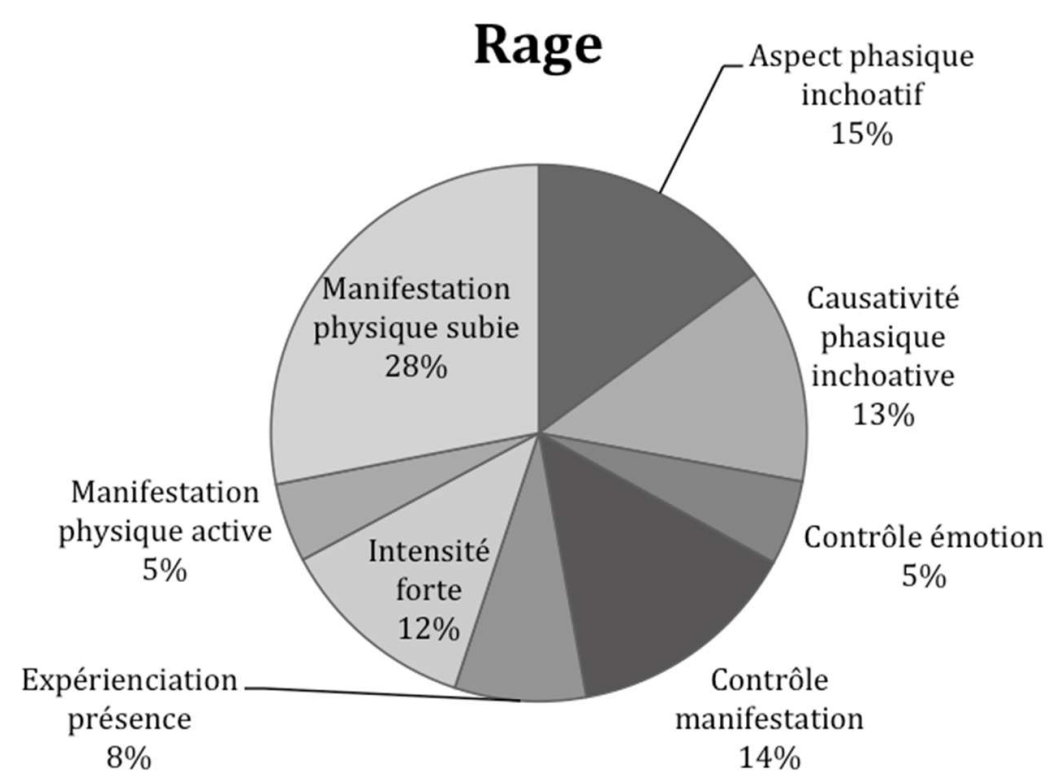

Figure 3 : Dimensions et valeurs sémantiques des collocatifs spécifiques de rage 
L'observation des dimensions et des valeurs sémantiques véhiculées par les collocatifs de fureur et de rage fait apparaitre des profils aux configurations distinctes : celui de fureur est largement dominé par une dimension (la causativité phasique inchoative, qui représente $60 \%$ des dimensions sémantiques des collocatifs de cette lexie), alors que dans celui de rage les dimensions sont réparties de façon plus homogène. Cependant, même si elles se manifestent dans des proportions différentes, fureur et rage partagent la plupart des dimensions et des valeurs sémantiques instanciées par leurs collocatifs :

- l'aspect, qui marque en l'occurrence la phase inchoative (la fureur s'empare de qqn, être saisi de rage) ;

- la causativité, qui marque également la phase inchoative et qui domine très nettement le profil de fureur (mettre qqn en rage/fureur, susciter/provoquer la fureur) ;

- le contrôle de la manifestation (une fureur/rage rentrée) ;

- l'intensité forte, pour laquelle on retrouve assez peu de collocatifs (fureur noire, vert de rage, une telle rage/fureur), l'affect étant déjà très intense, cette dimension se retrouve davantage au niveau textuel dans les réseaux isotopiques ;

- la manifestation physique subie, qui est la dimension dominante dans le profil de rage (trembler de rage, suffoquer de fureur).

Chacun des noms possède ensuite des dimensions sémantiques spécifiques, présentant néanmoins un poids collocationnel moindre, et n'étant presque toujours instanciées que par un seul collocatif. Ainsi, seul fureur possède des collocatifs exprimant la polaritéxiv. Il s'agit, en l'occurrence, d'une polarité notée «externe négative ${ }^{\mathrm{xv}}$ qui véhicule une évaluation portant sur l'affect (fureur sauvage). Cette évaluation axiologique (dimension sociale) pointe le manque de contrôle de l'affect en question (cf. Grutschus et al., 2013). Concernant les collocatifs aspectuels, nous n'en retrouvons également que pour fureur (aspect ponctuel itératif) avec fureurs soudaines. Rage, pour sa part, possède des collocatifs dénotant l'expérienciation, (c'est-à-dire le fait qu'une personne ressente un affect mais sans fournir d'information concernant la phase, la cause etc. comme dans la rage au ventre), mais aussi le contrôle de l'émotion (ravaler sa rage) et la manifestation physique active (lever, serrer le poing de rage).

\subsubsection{Les adjectifs rageur et furieux}

Les représentations graphiques des dimensions sémantiques des profils combinatoires de furieux et de rageur (figures 4 et 5) font apparaitre une situation bien différente de celles des noms dont ils sont dérivés. En effet, le nombre de dimensions sémantiques instanciées pour chacune de ces deux lexies est assez différent (sept dimensions pour furieux vs quatre pour rage), ce qui limite le recoupement sémantique. 


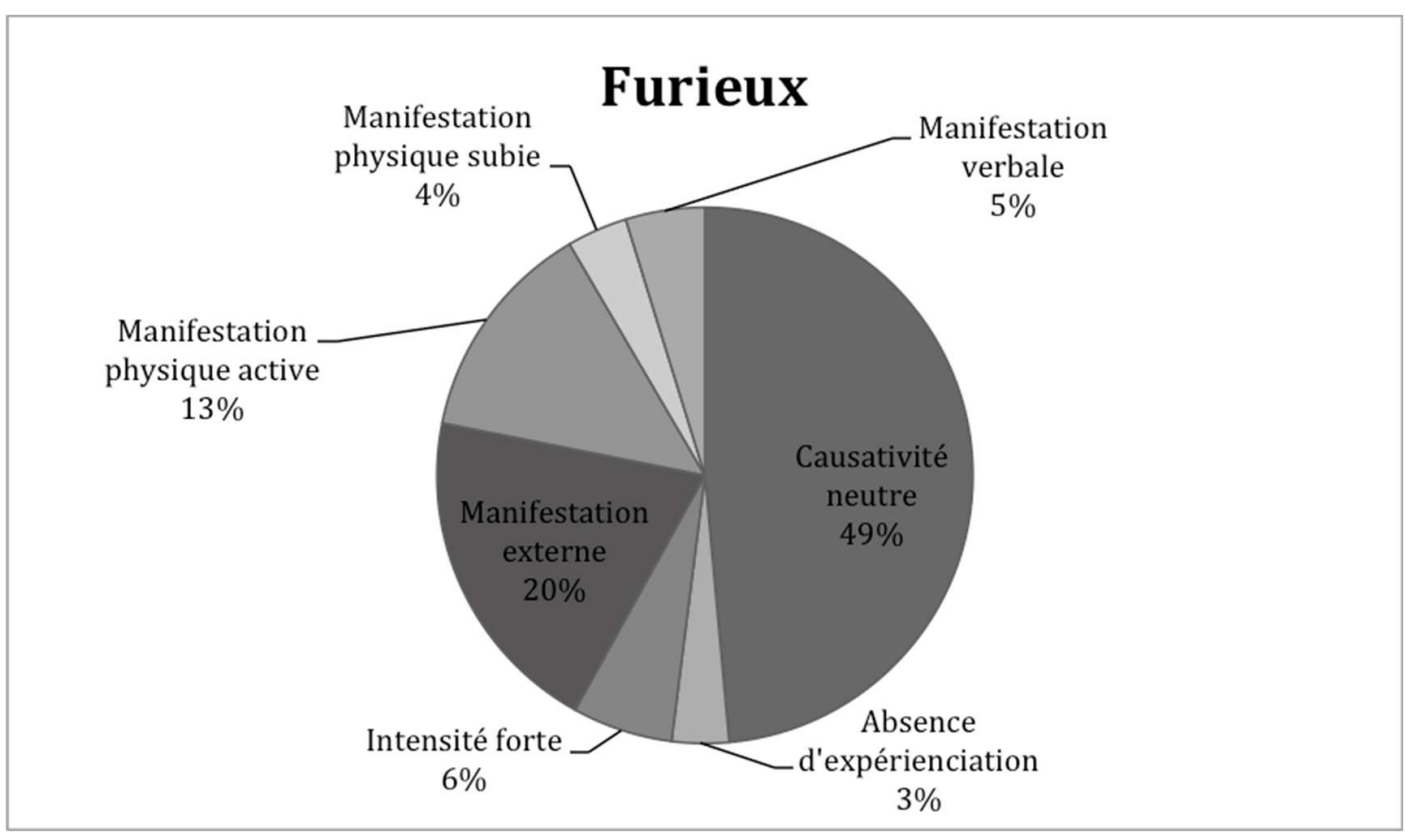

Figure 4 : Dimensions et valeurs sémantiques des collocatifs spécifiques de furieux

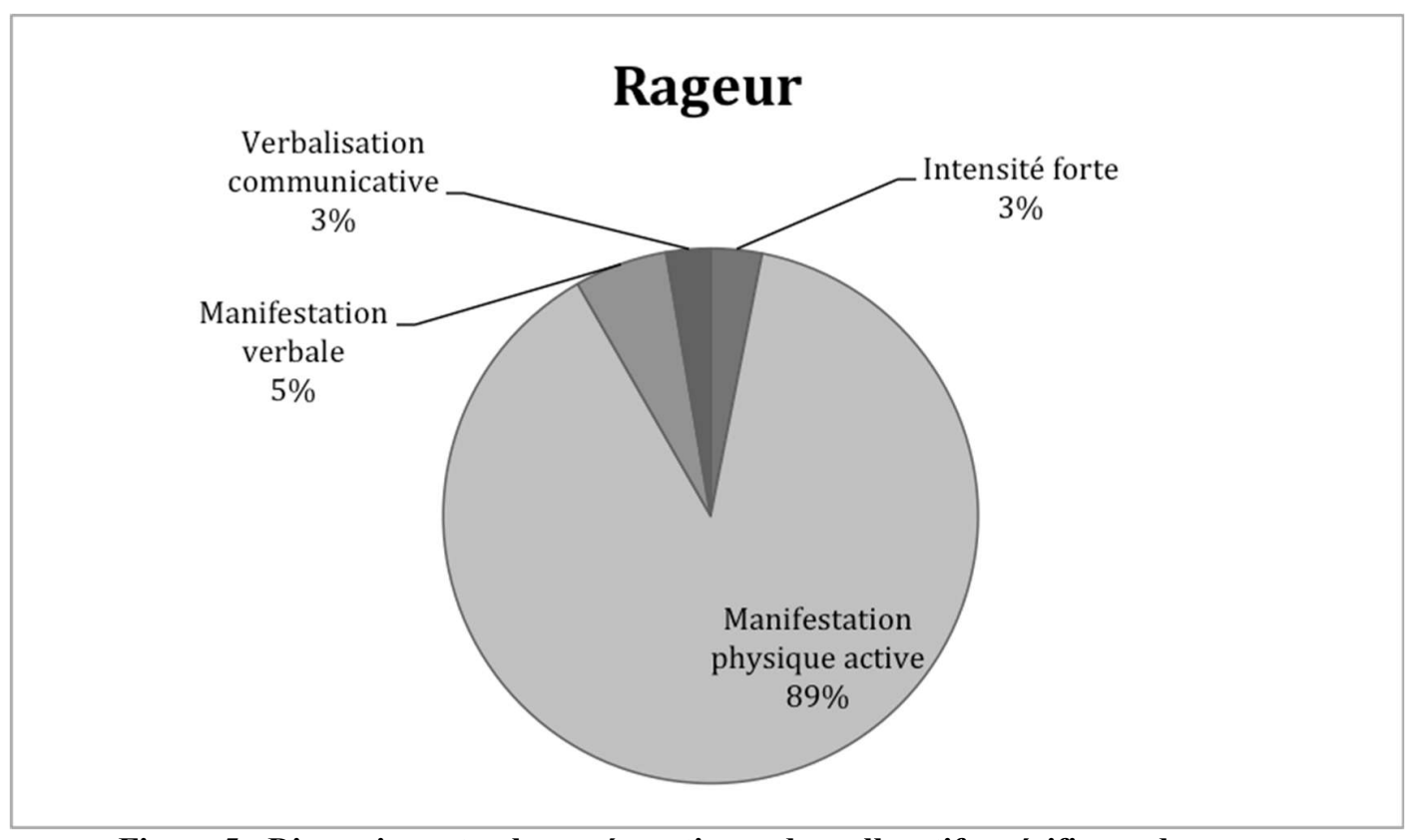

Figure 5 : Dimensions et valeurs sémantiques des collocatifs spécifiques de rageur 
Les couples dimensions/valeurs sémantiques communs à furieux et à rageur sont au nombre de trois :

- l'intensité forte, dans une faible proportion comme pour les noms (plus rageur/furieux);

- la manifestation verbale, dans une faible proportion également (ton rageur/furieux) ;

- la manifestation physique active, qui représente presque $90 \%$ du poids collocationnel du profil de rageur (geste rageur/furieux, regard furieux, coup de pied, poing, talon rageur).

Rageur n'a ainsi qu'une seule dimension sémantique qui lui soit spécifique (elle ne représente pour autant que 3\% du poids collocationnel) : la verbalisation, avec la valeur communicative $^{\mathrm{xvi}}$ (propos rageurs). Le profil de furieux est pour sa part dominé largement par une dimension sémantique qu'il ne partage pas avec rageur (mais qu'il partage avec fureur) : la causativité, en l'occurrence neutre. On n'y trouve cependant qu'une seule collocation, rendre furieux, pour laquelle le collocatif rendre possède une valeur de log-likelihood très élevée. Furieux possède également des collocatifs renvoyant au fait que l'affect en question n'est pas véritablement ressenti (absence d'expérienciation: presque furieux), ainsi qu'à des valeurs de la dimension manifestation qui ne sont pas instanciées pour rageur: manifestation externe (air furieux, paraître furieux, visiblement furieux) et physique subie (œil furieux).

\subsection{Associations sémantiques : les collocatifs spécifiques}

Après l'examen quantitatif des dimensions et des valeurs sémantiques véhiculées par les collocatifs de nos quatre lexies, nous analysons d'un point de vue qualitatif quels sont les collocatifs qui les instancient.

\subsubsection{Les collocatifs spécifiques des noms rage et fureur}

Le tableau 3 ci-dessous récapitule les différents collocatifs s'associant à fureur, à rage, ou aux deux lexies.

\begin{tabular}{|c|c|c|c|}
\hline & \multicolumn{2}{|c|}{ Fureur } & Rage \\
\hline $\mathbf{N}$ & & & $\begin{array}{r}\text { Larme, poing, } \\
\text { ventre }\end{array}$ \\
\hline $\mathbf{V}$ & $\begin{array}{l}\text { Provoquer, } \\
\text { susciter, } \\
\text { s'emparer, } \\
\text { suffoquer }\end{array}$ & Mettre & $\begin{array}{l}\text { Pleurer, trembler, } \\
\text { mouvoir, libérer, } \\
\text { ravaler, muer, } \\
\text { transformer, saisir }\end{array}$ \\
\hline
\end{tabular}




\begin{tabular}{|l|l|c|c|}
\hline $\mathbf{A}$ & $\begin{array}{l}\text { Noire, sauvage, } \\
\text { soudaine }\end{array}$ & $\begin{array}{c}\text { Plein de, telle, } \\
\text { rentrée }\end{array}$ & Vert de \\
\hline
\end{tabular}

Tableau 3 : Détail des collocatifs des noms fureur et rage

Nous pouvons tout d'abord remarquer une différence dans le type grammatical des collocatifs de fureur et de rage : il s'agit majoritairement d'adjectifs pour fureur $(6 / 11)$ et de verbes pour rage (9/16). Cette distribution est propre aux noms, une différence aussi nette n'est pas observable pour les adjectifs.

Concernant le sémantisme de ces collocatifs, rage et fureur partagent des collocatifs exprimant la causativité neutre, l'intensité et le contrôle de l'affect. Cependant, la majorité des collocatifs présents dans le tableau renvoie au début de l'affect, même si aucun n'est précisément commun aux deux noms. Cette dimension sémantique des collocatifs est dominante pour fureur, qu'elle soit associée ou non à la causativité : susciter, s'emparer, soudaine, etc. Pour rage, plusieurs collocatifs évoquent également le début de l'affect, mais avec des apports sémantiques spécifiques : muer, transformer, par exemple, dans des contextes où un sentiment généralement moins intense (colère, frustration, tristesse ...) se transforme en rage ${ }^{\text {xvii }}$. Enfin, on trouve pour rage beaucoup de collocatifs de manifestations physique actives ou subies (larme, poing, trembler, pleurer...), absents du profil de fureur.

\subsubsection{Les collocatifs spécifiques des adjectifs rageur et furieux}

Le tableau 4 ci-dessous récapitule les différents collocatifs s'associant à furieux, à rageur, ou aux deux lexies.

\begin{tabular}{|l|l|c|c|}
\hline & \multicolumn{2}{|c|}{ Furieux } & \multicolumn{2}{c|}{ Rageur } \\
\hline $\mathbf{N}$ & Regard, air, eeil & Ton, geste & $\begin{array}{l}\text { Propos, (coup de) } \\
\text { pied, poing, talon }\end{array}$ \\
\hline $\mathbf{V}$ & Rendre, paraître & & \\
\hline ADV & $\begin{array}{l}\text { Presque, } \\
\text { visiblement }\end{array}$ & Plus & \\
\hline
\end{tabular}

Tableau 4 : Détail des collocatifs des adjectifs furieux et rageur

Il apparaît que rageur est d'emploi plus figé, du fait de l'absence de collocatifs autre que nominaux. Du point de vue sémantique, l'intensité fait partie, comme pour les noms, des dimensions sémantiques partagées par les deux adjectifs. On constate également deux collocatifs communs, qui traduisent une manifestation dans le cadre d'une relation interpersonnelle: ton et geste. Du point de vue des collocatifs spécifiques à chaque adjectif, on trouve, pour rage comme pour fureur, des collocatifs qui renvoient à des parties du corps qui sont cependant nettement différentes en fonction de l'adjectif auquel elles sont associées. Rageur se combine ainsi avec des 
noms renvoyant aux extrémités du corps (pied, poing, talon) et susceptibles de manifester l'affect de façon volontaire (la manifestation physique active domine largement le profil de rageur, comme nous l'avons montré dans la section précédente). Furieux, quant à lui, s'associe préférentiellement à des noms dénotant des parties ou des propriétés du visage (regard, air, ail), susceptibles de révéler ou de marquer l'affect. Enfin, furieux présente plusieurs collocatifs qui expriment un jugement externe sur l'existence et l'intensité de l'affect d'autrui : visiblement, paraître..., ce qui contraste avec la prédominance des manifestations physiques actives propres à rage.

L'analyse des associations sémantiques fait ainsi émerger des caractéristiques propres à chaque nom et adjectif des couples de lexies étudiées. Ainsi, fureur et furieux s'associent principalement à des collocatifs exprimant la causativité, alors que rage et rageur privilégient les associations avec des collocatifs exprimant des manifestations, et plus particulièrement des manifestations physiques actives. Ces premiers constats, qui tendent à rapprocher le nom et l'adjectif dont il est dérivé, sont néanmoins à relativiser car l'analyse de leur profil syntaxique oppose les deux couples nom-adjectif.

\section{Profil syntaxique}

Le profil syntaxique des lexies s'élabore par l'analyse de trois phénomènes. Nous commencerons notre étude par la description des configurations actancielles en utilisant le codage conventionnel des actants sémantiques ${ }^{\text {xviii }}$ qui distingue :

- X: l'expérienceur de l'affect, c'est-à-dire celui qui éprouve la rage ou la fureur ;

- Z : la cause de l'émotion, c'est-à-dire ce qui provoque la rage ou la fureur ;

- Y : l'objet de l'émotion, c'est-à-dire ce vers quoi ou ce vers qui se manifeste la rage ou la fureur.

Dans un deuxième temps, nous examinerons la nature de l'expérienceur. En effet, il est des cas où l'expérienceur de l'affect $(\mathrm{X})$ réfère à une entité non humaine. Nous essayerons de voir si ce facteur peut être considéré comme discriminant dans le profil de chacune des lexies. Enfin, nous analyserons les positions et les fonctions grammaticales occupées par les unités lexicales étudiées.

\subsection{Les configurations actancielles}

Les quatre lexies étudiées peuvent se rencontrer au sein de quatre configurations actancielles en fonction de l'absence (emploi avalent) ou de la présence d'un, de deux ou de trois actants sémantiques (emplois monovalent, bivalent ou trivalent). 


\subsubsection{Les configurations actancielles de rage et de fureur}

a. Emplois monovalents

L'immense majorité des emplois monovalents de rage et de fureur instancie l'expérienceur (X), comme dans les exemples (1) et (2) :

(1) Mais elle (X) s'est avancée vers moi, pleine d'une rage froide qui m'a fait peur. (J.M.G. Le Clézio, Poisson d'or)

(2) Et puis brusquement vous (X) voilà en proie à une noire fureur. (F. Dard, Chez les macs)

Notre corpus ne comporte qu'une seule occurrence où l'actant sémantique cause $(Z)$ est instancié :

(3) Sans la fureur provoquée par M. Summers (Z), le Pr Faust aurait-elle été choisie pour diriger Harvard? (Le Monde, 2007).

L'emploi monovalent avec instanciation seule de l'expérienceur (X) se présente comme l'emploi prototypique des collocations de la lexie rage $(60 \%)$, alors que cette structure ne concerne qu'un tiers des occurrences de fureur.

b. Emplois bivalents

Dans les emplois bivalents, la seule configuration attestée pour les lexies rage et fureur est l'instanciation conjointe de l'expérienceur (X) et de la cause (Z). Aucune occurrence ne présente le cas de figure où l'on aurait la combinaison de l'expérienceur $(\mathrm{X})$ et de l'objet (Y), ou bien celle de la cause (Z) et de l'objet (Y). Cette configuration, qui représente plus des deux-tiers des occurrences de fureur (essentiellement avec le collocatif provoquer), n'est pas surprenante vu le poids des collocatifs causatifs (cf. supra) :

(4) La confusion (Z) a-t-elle provoqué la fureur des Allemands (X) ? (Le Figaro, 2008)

Dans le cas de rage, la structure bivalente se rencontre dans un tiers des collocations de la lexie, essentiellement avec le collocatif causatif mettre :

(5) Son échec $(\mathrm{Z})$ lui $(\mathrm{X})$ avait mis la rage au cour $(\mathrm{P} / \mathrm{X})$. (M. Druon, Les rois maudits)

Dans l'exemple (5), le nom coeur désigne une partie de l'expérienceur (X), précisément le siège où opère l'affect. En suivant la terminologie du DEC (Dictionnaire explicatif et combinatoire du français contemporain: recherches lexico-sémantiques, 19841999), on pourrait y voir une « propriété » ou une « composante » de (X) dans laquelle 
se manifeste le fait que (X) est en rage. Nous proposons ainsi de la coder $(\mathrm{P} / \mathrm{X})$, dans la lignée de ce qui a été observé pour les verbes d'affect (Novakova, Grossmann \& Goossens, 2013b) où l'on trouve des dédoublements de la cause $(\mathrm{P} / \mathrm{Z})$ ou de l'objet $(\mathrm{P} / \mathrm{Y})$. Apparait ici en l'occurrence un dédoublement de l'expérienceur. Nous considérons comme actant sémantique, au niveau phrastique, non pas la partie dans laquelle se manifeste l'affect $(\mathrm{P} / \mathrm{X})$, mais le tout qui l'éprouve $(\mathrm{X})$. L'étiquette $(\mathrm{P} / \mathrm{X})$ ne désigne donc pas un actant sémantique à lui tout seul. L'emploi bivalent avec instanciation de l'expérienceur $(X)$ et de la cause $(Z)$ se présente comme l'emploi prototypique des collocations de la lexie fureur (70\%), alors qu'il ne couvre qu'un tiers des emplois de rage.

c. Emplois trivalent et avalent de rage

Les deux autres configurations actancielles attestées, trivalentes et avalentes, sont marginales. En effet, notre corpus ne révèle qu'un seul exemple (6) dans lequel une collocation de rage instancie trois actants sémantiques, l'expérienceur $(\mathrm{X})$, la cause $(\mathrm{Z})$ et l'objet (Y) :

(6) Je ne sais pas ce qu'elle fabrique, je ne sais même pas si elle l'a fait exprès, mais ça $(\mathrm{Z})$ me $(\mathrm{X})$ met en rage contre elle, contre moi $(\mathrm{Y})$ ! (P. Lemaitre, Robe de marié)

La réalisation complète des actants sémantiques est plus coûteuse que le simple 'je suis en rage'. Néanmoins, elle se comprend dans le cadre d'une stratégie argumentative où l'émotion est mise en scène dans le discours. En effet, le déploiement d'une structure à trois actants est utilisé, au même titre que les parallélismes de construction (je ne sais pas [...] je ne sais pas; contre elle, contre moi) comme un élément du procédé de répétition. L'insistance ainsi instaurée fait partie des techniques de persuasion et plus généralement du recours au pathos (Micheli, 2010, p. 43-56).

Enfin, quelques occurrences sans instanciation d'aucun actant sémantique au niveau phrastique se rencontrent parfois avec des collocations de rage. Le recours au niveau transphrastique est alors nécessaire pour actualiser les actants sémantiques :

(7) Alors Maud a eu peur, seule dans le noir, sur cette mer démontée avec son mât qui frappait la coque tel un bélier en furie. À ce moment-là, Maud a pensé à abandonner. La peur et la rage au ventre, intimement liées, ennemies inséparables. Et puis le jour est venu. (Le Figaro 2007)

(8) Le mâle fait un malheur! Il fonce, il cogne! La rage aux poings, la hargne au cour, la grogne aux pieds ! (F. Dard, Béru-Béru)

Ces rares emplois avalents apparaissent majoritairement au sein de phrases averbales. L'effacement des actants sémantiques au niveau phrastique contribue, par son caractère 
compact, à la stratégie rhétorique, au même titre que les autres procédés utilisés tels le rythme binaire des séquences (7) ou l'énumération (8). La possibilité de rencontrer les collocations de rage dans des emplois trivalents ou avalents contribue ainsi à singulariser la lexie par rapport à son synonyme fureur.

Pour résumer, les emplois avalents et trivalents sont très minoritaires pour rage et inexistants pour fureur. Les deux configurations attestées majoritairement sont les structures monovalente et bivalente. En outre, les proportions d'emplois monovalents et bivalents sont inversées pour les deux lexies : rage se rencontre surtout en emploi monovalent (60\%) alors que la configuration bivalente domine pour fureur (70\%). Les deux lexies ont donc bien deux profils actanciels distincts.

\subsubsection{Les configurations actancielles de furieux et de rageur}

a. Emplois bivalents

Cette configuration se réalise pour rageur (9) et pour furieux (10) par l'instanciation des deux actants sémantiques : l'expérienceur (X) et la cause (Z).

(9) Il (X) chassa d'un geste $(\mathrm{P} / \mathrm{X})$ rageur quelques gamins venus quémander des piécettes $(\mathrm{Z})$. (M. Houellebecq, Plateforme)

(10) «Ils (Z) me (X) rendent furieux », confie l'écrivain. (Le Figaro, 2007)

Dans l'exemple (9), le nom geste, dont l'adjectif rageur est épithète, désigne, par le biais d'une relation méronymique, l'expérienceur (X). Il s'agit ici encore d'un cas de dédoublement de l'expérienceur $(\mathrm{P} / \mathrm{X})$. Les emplois bivalents représentent la configuration prototypique pour les collocations avec rageur, alors qu'elle est beaucoup plus rare pour furieux puisqu'elle apparait uniquement avec trois collocatifs (regard, ton, rendre).

$\mathrm{Au}$ sein des emplois bivalents apparait une configuration commune à rageur et furieux pour deux de leurs collocatifs : coup de poing et poing pour rageur, ton et rendre pour furieux.

(11) Le dromadaire laisse retomber son pied sur celui de Beru (Z), lequel (X) étant chaussé de babouches, pousse un hurlement et donne un coup de poing $(\mathrm{P} / \mathrm{X})$ rageur dans les flancs de l'animal (lieu). (F. Dard, Bérurier au sérail)

(12) Rendue furieuse par une longue réclusion (Z), une vipère noire (X) bondit pour mordre sa victime au cou (lieu). (C. Jacq, La pierre de lumière)

C'est un phénomène saillant et inédit qui vient instaurer un expérienceur $(X: B e ́ r u, ~ l a$ vipère noire), une cause de l'affect ( $\mathrm{Z}$ : le dromadaire qui écrase le pied de Béru, la longue réclusion de la vipère) et un troisième élément qui indique le lieu où 
s'externalise l'affect (les flancs du dromadaire, le cou de la victime), noté (lieu). Dans les deux cas $(11,12)$, la mise en valeur de la cause $(Z)$, en position antéposée à l'expérienceur $(\mathrm{X})$, permet de réaliser un centrage discursif sur la cause de l'affect (cf. Fesenmeier, 2010).

b. Emplois monovalents

À l'inverse, les emplois monovalents constituent la configuration la plus fréquente au sein de laquelle apparaissent furieux et ses collocatifs :

\section{(13) Lorenzo (X) a l'air furieux. (P. Cintas, Carabin Carabas)}

Dans la quasi-totalité des occurrences, l'expérienceur (X) est le seul actant sémantique instancié comme en (13). Cette configuration est extrêmement rare pour rageur (5\%).

c. Emplois avalents

À l'instar du nom rage, l'adjectif rageur se rencontre parfois dans des configurations avalentes :

(15) Ouvrez, on sait que vous êtes là ! Le préposé (X) muni du filet à vieux cognait à grands coups dans la porte d'entrée. Ils se pelotonnèrent l'un contre l'autre. [Aux coups de poing $(\mathrm{P} / \mathrm{X})$ rageurs succédèrent les coups de pied.] Dans leur cage grillagée, les Foultrant baissaient la tête. (B. Werber, L'arbre des possibles)

(16) Poings (P/X) rageurs. (Libération, 2007)

Dans l'exemple (15), c'est uniquement au niveau transphrastique qu'est instancié l'actant sémantique $(\mathrm{X})$, le préposé. Son effacement dans la phrase contribue, au même titre que les autres procédés stylistiques (hyperbate avec antéposition du complément, répétition lexicale), à amplifier la brutalité de l'affect décrit. Dans l'exemple (16), l'intertitre journalistique "Poings rageurs » met en œuvre une stratégie rhétorique banale qui consiste à provoquer un effet d'attente chez le lecteur par la sécheresse d'un énoncé averbal sans actant sémantique exprimé.

Pour résumer, les emplois avalent et trivalent sont très minoritaires pour rageur et furieux. De la même manière que pour les noms rage et fureur, les deux configurations attestées majoritairement sont les structures monovalentes et bivalentes. Néanmoins, les proportions d'emplois prototypiques sont inversées par rapport aux deux noms correspondants : rageur se rencontre majoritairement en emploi bivalent alors que la configuration monovalente domine pour furieux. Les deux lexies ont non seulement deux profils actanciels distincts, mais ils diffèrent en outre de ceux des noms correspondants. 


\subsection{Nature de l'expérienceur (X)}

Dans le cadre de l'étude des structures actancielles de noms d'affect, nous avons montré que l'expérienceur de l'affect $(\mathrm{X})$ ne réfère pas explicitement à un être humain (cf. supra (12) où (X) est une vipère noire). C'est ainsi que la fureur ou la rage peuvent s'emparer non seulement d'un taureau ou d'un étalon (expérienceur (X) animé), mais également d'un quartier, d'une bataille, d'une histoire, d'un pays, d'une maison de disques, des mains ou encore d'un récit (expérienceur (X) inanimé), comme dans l'exemple suivant :

(17) D'autres personnages surgiront au fil d'un récit (X) qui tourne les pages des destins d'un geste rageur. (Libération, 2007)

L'attribution d'un affect à un inanimé entre dans le cadre d'une stratégie rhétorique par création de figures : dans l'exemple (17), il s'agit d'une personnification, ailleurs on trouve des métonymies du lieu ou des parties du corps. Ce procédé est nettement dominant dans la partie journalistique de notre corpus $(60 \%)$. La présence d'un expérienceur (X) non humain est davantage attestée avec les collocations de fureur/furieux (70\%) qu'avec celles de rage/rageur (30\%). La possibilité d'instancier un expérienceur de l'affect non humain corrobore l'idée que les lexies rage/rageur et fureur/furieux sont des noms d'affect un peu particuliers en se révélant proches des noms d'attitude, ou de comportement (Anscombre, 1995, 1996 ; Buvet et al., 2005) : les animaux peuvent éprouver ou en tout cas manifester de la fureur et de la rage, plus difficilement de la nostalgie ou de la commisération.

\subsection{Fonctions récurrentes dans la phrase}

Selon la théorie du Lexical priming de Hoey (2005), les mots ont des préférences ou des aversions pour certaines positions, et de là, pour des fonctions grammaticales données (ou « colligations »). Nous allons donc examiner la distribution des lexies au sein des collocations relevées.

\subsubsection{Fonctions récurrentes de fureur et de rage}

Les deux lexies fureur et rage se rencontrent dans trois fonctions grammaticales différentes : complément prépositionnel, complément direct et sujet. Néanmoins, les préférences d'emploi diffèrent de l'une à l'autre. En effet, la construction prépositionnelle est privilégiée pour rage ( $75 \%$ : être saisi de rage, trembler de rage), nettement moins pour fureur ( $43 \%$ : plein de fureur). En revanche, fureur se rencontre 
dans presque la moitié de ses occurrences en position de complément direct ( $46 \%$ : provoquer la fureur, susciter la fureur), alors que cet emploi se révèle plus rare pour rage (16\% : libérer sa rage, ravaler sa rage). Le poids des collocatifs causatifs plus marqué pour fureur explique cette situation. Enfin, les deux lexies sont très peu employées en position de sujet (6\% pour rage, $10 \%$ pour fureur : la fureur s'empare de, la rage se transforme).

\subsubsection{Fonctions récurrentes de furieux et de rageur}

Les deux adjectifs furieux et rageur apparaissent au sein de GN occupant les fonctions de complément prépositionnel ou de complément direct. Même si les deux lexies ont en commun le fait d'être employées comme qualificatif unique du nom qu'elles accompagnent, les fonctions privilégiées par l'une et l'autre diffèrent. La construction prépositionnelle est l'emploi prototypique des collocations de rageur (62\%), la construction standard se présentant sous la forme de $+\mathrm{N}+$ rageur (d'un geste $\sim$, d'un ton $\sim$, d'un poing $\sim$, etc. $)^{\text {xix }}$. Pour sa part, furieux apparait deux fois moins souvent dans ce type de constructions ( $35 \%:$ d'un ton $\sim$, d'un air $\sim$, d'un æeil $\sim$ etc.) et se distingue en outre par la grande variété des prépositions qui introduisent le GN dans lequel il se trouve (de, avec, sous, par, sur, dans). L'emploi privilégié par les GN où il apparait est nettement celui de complément direct (50\% : lancer un regard furieux, avoir un air furieux), celui-ci étant deux fois moins fréquent pour les collocations de rageur $(22 \%$ : lever un poing rageur, voir un geste rageur, décocher un coup de pied rageur).

\section{Profil textuel}

L'élaboration du profil textuel repose sur l'analyse de phénomènes dépassant le cadre de la phrase. En nous demandant si le genre des textes pouvait être un facteur influent dans le choix des lexies, nous avons pu observer une répartition sensiblement égale des lexies dans les deux sous-corpus, littéraire (L) et journalistique (J) : fureur $58 \%(\mathrm{~J})$ $42 \%(\mathrm{~L})$, furieux $50 \%$ - 50\%, rageur 52\% (J) - 48\%(L), à l'exception notable de rage, $33 \%(\mathrm{~J})-57 \%(\mathrm{~L})$. Néanmoins, comme le sous-corpus littéraire contient six fois moins de mots que le sous-corpus journalistique, nous pouvons seulement conclure que les noms d'affect sont davantage employés dans le corpus littéraire que dans le journalistique. Ce phénomène corrobore ce qu'a déjà mis en lumière une autre étude menée sur deux sous-corpus parallèles équilibrés (cf. Goossens 2011) ${ }^{\mathrm{xx}}$. Nous avons également examiné les registres de langue pour tenter de mettre en évidence une spécificité du profil textuel de l'une ou de l'autre des lexies. L'analyse des occurrences révèle que ces quatre lexies se rencontrent au sein de collocations apparaissant aussi bien dans les œuvres de F. Dard, dont les dialogues sont émaillés d'expressions 
populaires et régionales, que dans celles de l'académicien M. Druon. Il n'est donc pas possible de conclure sur l'accointance privilégiée de telle ou telle lexie avec un registre de langue spécifique.

L'examen des réseaux isotopiques ${ }^{x x i}$ offre des pistes plus fécondes. On pourrait supposer que plus on s'éloigne de l'occurrence du mot pivot, plus la liberté de choix des cooccurrents est grande. Or, certaines unités lexicales sont plus prévisibles que d'autres dans l'environnement textuel de la lexie. En effet, la récurrence des isotopies de l'intensité et de la polarité négative est importante dans l'environnement textuel des collocations de furieux et de fureur:

(18) De plus, l'écart entre les taux d'intérêt américain et européen - qui explique une partie de la flambée de l'euro vis-à-vis du dollar car les capitaux affluent vers une zone euro plus rémunératrice - devrait s'amplifier. [...]. Ces éléments préfigurent-ils d'une chute sévère à venir du dollar? [...]. C'est en tout cas le scénario que craint le directeur général du Fonds monétaire international, Rodrigo Rato, qui a déclaré, lundi, que "le risque d'une chute brutale du dollar existe », conséquence ou cause "d'une perte de confiance dans les actifs libellés dans la monnaie américaine ». Ces propos ont rendu furieux les responsables politiques et monétaires, qui se seraient bien passés d'une telle marque de pessimisme. (Le Monde, 2007)

Ces deux isotopies sont actualisées conjointement et très majoritairement dans le cas des collocations de furieux et de fureur (réseaux isotopiques de l'intensité avec flambée, s'amplifier, sévère, brutale, et de la polarité négative avec chute, risque, craint, perte de confiance, pessimisme). Cette caractéristique du profil textuel des deux lexies est partagée avec d'autres noms d'affect comme stupeur et jalousie par exemple (cf. Novakova \& Sorba, 2013b, 2014).

Pour sa part, l'environnement textuel des collocations de rage et de rageur actualise régulièrement (25\%) une isotopie de la polarité positive, en plus de celle de l'intensité :

(19) Au fil des jeux, Kutznetsova trouvait quelques repères. Réussissant, au moins pour un temps, à prendre le dessus en faisant durer les échanges, elle s'emparait de nouveau du service de Safina pour revenir à 4-3. Mais il n'en fallait pas plus pour piquer au vif la récente gagnante du tournoi de Berlin. Safina empochait la première manche 6-3, poing rageur après avoir réalisé une jolie amortie gagnante. Déterminée, Safina se détachait dans la deuxième manche (6-2) pour s'envoler vers sa première finale d'un tournoi majeur. Un succès au terme d'une rencontre décousue face à une Kuznetsova qui n'était pas dans un bon jour. (Le Figaro, 2008) 
Les réseaux isotopiques de l'intensité (prendre le dessus, piquer au vif, majeur) et de la polarité positive (réussissant, gagnante, jolie, succès) sont riches et variées. Dans ce cas, la collocation poing rageur apparait toujours dans la rubrique « sport» du corpus journalistique. Les scènes de victoire qui y sont décrites représentent ce que Plantin nomme des «situations culturellement formatées pour une émotion» $(2012,367)$. Ainsi, la présence du réseau isotopique de la polarité positive répond à l'attente précontrainte de ce type d'article ${ }^{\mathrm{xxii}}$. C'est ainsi que nous pouvons expliquer la neutralisation de la polarité négative héritée par défaut dans rage/rageur. La présence régulière de l'isotopie de la polarité positive permet alors de distinguer le profil textuel de rageur de celui furieux.

Néanmoins, l'isotopie tissée autour de la lexie peut aussi s'avérer purement de circonstance, comme pour la collocation vert de rage. En effet, le réseau isotopique actualise systématiquement au moins un référent de la couleur verte :

(20) Vert de rage en ce mois de mai Patrick Stutzinger, de Guichen, revient sur des événements qui ont fait l'actualité localement ces derniers temps. "Alors que les mots écologie et développement durable se conjuguent maintenant à toutes les sauces, ce mois de mai, riche en allergies, me donne quelques nausées. »(Ouest-France, 2007)

Dans l'exemple (20), les associations avec la couleur verte sont de l'ordre symbolique mais bien réelles, le mois de mai évoquant le printemps verdoyant et les mots écologie et développement durable étant associés à cette couleur dans les représentations collectives culturelles françaises.

\section{Synthèse}

Une synthèse du profil discursif des quatre lexies met en évidence les différences de comportement combinatoire, avec tour à tour les spécificités discriminantes de la paire ragelfureur (tableau 5), puis celles du couple rageurlfurieux (tableau 6).

\begin{tabular}{|c|c|c|}
\hline & Rage & Fureur \\
\hline Dimension sémantique dominante & $\begin{array}{l}\text { Manifestation } \\
\text { physique }\end{array}$ & Causativité \\
\hline Type de collocatifs privilégié & Verbal & Adjectival \\
\hline Structure actancielle dominante & Monovalente & Bivalente \\
\hline $\begin{array}{l}\text { Structure(s) } \\
\text { spécifique(s) }\end{array}$ & Trivalente, avalente & $\varnothing$ \\
\hline
\end{tabular}




\begin{tabular}{|l|l|l|}
\hline Fonction syntaxique privilégiée & GP complément & $\begin{array}{l}\text { GN complément } \\
\text { direct }\end{array}$ \\
\hline Réseaux isotopiques dominants & $\begin{array}{l}\text { Intensité, polarité } \\
\text { positive }\end{array}$ & $\begin{array}{l}\text { Intensité, polarité } \\
\text { négative }\end{array}$ \\
\hline
\end{tabular}

Tableau 5 : Les différences de comportement combinatoire pour la paire rage/fureur

\begin{tabular}{|c|c|c|}
\hline & Rageur & Furieux \\
\hline Dimension sémantique dominante & $\begin{array}{l}\text { Manifestation } \\
\text { physique }\end{array}$ & Causativité \\
\hline Type de collocatifs privilégié & Nominal & Nominal \\
\hline Association lexicale remarquable & $\begin{array}{ll}\begin{array}{l}\text { Partie du corps } \\
\text { (extrémités) }\end{array} & \\
\end{array}$ & $\begin{array}{lll}\begin{array}{l}\text { Partie } \\
\text { (visage) }\end{array} & \text { du } & \text { corps } \\
\end{array}$ \\
\hline Structure actancielle dominante & Bivalente & Monovalente \\
\hline Fonction syntaxique privilégiée & GP complément & $\begin{array}{ll}\mathrm{GN} & \text { complément } \\
\text { direct } & \\
\end{array}$ \\
\hline Réseaux isotopiques dominants & $\begin{array}{l}\text { Intensité, polarité } \\
\text { positive }\end{array}$ & $\begin{array}{l}\text { Intensité, polarité } \\
\text { négative }\end{array}$ \\
\hline
\end{tabular}

Tableau 6 : Les différences de comportement combinatoire pour la paire rageur/furieux

Les profils lexicaux montrent que les noms fureur et rage ont chacun une dimension sémantique différente très notable: la causativité pour le premier, la manifestation physique active pour le second. Cette répartition se retrouve avec les adjectifs correspondants, la dimension manifestation physique active étant toutefois encore plus marquée pour rageur que pour rage. D'autre part, les lexies se distinguent entre elles par le type grammatical de leurs collocatifs spécifiques : rage se rencontre surtout au sein de collocations verbo-nominales (pleurer de rage) alors que ce sont les collocatifs de type adjectival qui prédominent pour fureur (fureur noire). De plus, au sein des collocations, les adjectifs dérivés se répartissent nettement la qualification des parties du corps : à rageur sont associés les noms des extrémités alors que ce sont les noms des partie du visage qui sont qualifiées de furieux.

En outre, les profils syntaxiques révèlent que les emplois monovalents dominent pour le nom rage, alors que la structure actancielle prototypique de fureur est bivalente. En revanche, pour les adjectifs, c'est l'inverse: furieux entre majoritairement dans des structures actancielles monovalentes, tandis que rageur apparait davantage en emplois bivalents. Les structures actancielles prototypiques des noms et des adjectifs correspondants, même si elles ne sont pas identiques, permettent néanmoins de discriminer les synonymes entre eux. Nous avons relevé également une particularité récurrente du couple fureur/furieux qui peut posséder un expérienceur non humain, alors que c'est peu fréquent dans le cas de rage/rageur. Le profil syntaxique révèle un dernier point pertinent dans notre objectif de différenciation des synonymes 
grâce à l'étude des fonctions syntaxiques, puisque les couples nom/adjectif ont chacun une construction privilégiée différente (au sein de GP pour rage/rageur, au sein de GN complément direct pour fureur/furieux).

Enfin, l'étude des réseaux isotopiques montre que l'intensité est omniprésente dans l'environnement textuel des lexies. Cette dimension sémantique, qui apparait peu dans la combinatoire avec les collocatifs, est quasi systématiquement présente au niveau transphrastique. Néanmoins, alors que le groupe furieux/fureur se distingue par une actualisation conjointe de l'isotopie de la polarité négative, les collocations de rage/rageur se rencontrent régulièrement dans un réseau lexical tissant l'isotopie de la polarité positive.

\section{Conclusion}

L'étude de corpus présentée a permis d'établir le profil discursif des lexies rage/rageur et fureur/furieux en analysant, au sein de leurs collocations, les moyens linguistiques (structures actancielles, associations lexicales véhiculant différentes dimensions sémantiques, réseaux isotopiques) mis au service de l'argumentation et de la cohésion textuelle, et ce, aussi bien au niveau microtextuel (dans le cadre de la phrase) que macrotextuel. L'application de l'analyse combinatoire sur un vaste corpus montre ainsi son intérêt tant pour différencier des lexies entre elles que pour les situer plus largement au sein de la classe des lexies d'affect. Nous avons pu observer, d'une part, que les lexies rage/rageur et fureur/furieux, généralement présentées par les outils lexicographiques comme des synonymes, se distinguent en réalité sur bien des points au sein de leurs collocations respectives. D'autre part, l'analyse de la combinatoire a également permis de rapprocher les membres de ces deux couples, en mettant en évidence leur caractère proche des lexies d'attitude: présence fréquente de manifestation, dédoublement de l'expérienceur et expérienceur pouvant être non humain etc. Enfin, notre étude a permis de montrer que ces distinctions comme ces regroupements se manifestent au sein de la phrase, mais également au niveau macrotextuel qui est, à ce jour, plus rarement étudié de manière systématique.

\section{Références bibliographiques}

Anscombre, J.-C. (1995). Morphologie et représentation événementielle : le cas des noms de sentiment et d'attitude. Langue Française, 105, pp. 40-54.

Anscombre, J.-C. (1996). Noms de sentiment, noms d'attitude et noms abstraits. In N. Flaux, M. Glatigny, \& D. Samain (Eds.), Les noms abstraits. Histoire et théories (pp. 257-273). Lille : Presses Universitaires du Septentrion. 
Baider, F. \& Constantinou, M. (2014a). Scénario cognitif et 'mises en scènes' des émotions : étude contrastive des concepts de colère et de rage en grec et en français. In P. Blumenthal, I. Novakova \& D. Siepmann (Eds.), Les émotions dans le discours. Emotions in discourse (pp. 55-67). Frankfurt am Main : Peter Lang.

Baider, F. \& Constantinou, M. (2014b). La fureur de gagner, la rage de perdre. Étude contrastive de colère, de rage et de fureur en français et en grec moderne. In J. Sorba, \& C. Cusimano (Eds.), Sens multiple(s) et polysémie. Regards d'Occident (pp. 89-104). Études Romanes de Brno n³5.1.

Baider, F. \& Cislaru, G. (Eds.). Cartographie des émotions. Propositions linguistiques et sociolinguistiques. Paris : Presses de la Sorbonne Nouvelle.

Blumenthal, P. (2002). Les péchés capitaux : profil combinatoire et dimension textuelle. In M. Kesik (Ed.), Référence discursive dans les langues romanes et slaves (pp.29-45). Lublin : Wydawnictwo Uniwersytetu Marii Curie-Skłodowskiej.

Buvet, P.-A., Girardin, C., Gross, G., \& Groud, C. (2005). Les prédicats d'affect. Lidil, 32, pp. 123-143.

Blumenthal, P. (2007). Sciences de l'Homme vs sciences exactes : combinatoires des mots dans la vulgarisation scientifique. Revue française de linguistique appliquée, 12(2), pp.15-28.

Deignan, A. (1999). Corpus-based research into metaphor. In C. Lynne \& L. Graham (Eds.), Researching and Applying Metaphor (pp.177-199). Cambridge : CUP.

Dunning T. (1993). Accurate methods for the statistics of surprise and coincidence. Computational Linguistics, 19(1), pp.61-74.

Fesenmeier, L. (2010). "Se souvenir" en français et en italien : différence(s) de centrage. In M. Iliescu, H. Siller-Runggaldier \& P. Daniel (Eds.), Actes du XXV Congrès International de Linguistique et de Philologie Romanes, tome III, section 7 (pp. 85-96). Tübingen : Niemeyer.

Goossens, V. (2005). Les noms de sentiment : esquisse de typologie sémantique fondée sur les collocations verbales. Lidil, 32, pp.103-127.

Goossens, V. (2011). Propositions pour le traitement de la polysémie régulière des noms d'affect. Thèse de doctorat. Université de Grenoble - LiDiLEM.

Gross, M. (1981). Les bases empiriques de la notion de prédicat sémantique. Langages, 63, pp.7-52.

Gross, M. (1995). Une grammaire locale de l'expression des sentiments. Langue française, 105 , pp.70-87.

Grossmann, F. \& Tutin, A. (2003). Les collocations. Analyse et traitement. Travaux de recherches en linguistique appliquée. Amsterdam : De Werelt.

Grutschus, A., Kern, B. \& Tutin, A. (2013). La polarité du lexique de l'affect : perspective combinatoire et contrastive. In G. Cislaru \& F. Baider (Eds.), Cartographie des émotions. Propositions linguistiques et sociolinguistiques (pp. 85-96). Paris : PSN.

Hoey, M. (2005). Lexical priming. A New Theory of Words and Language. Londres - New York : Routledge.

Imbs, P. \& Quemada, B. (Eds.) (1994). Trésor de la Langue Française. [version informatisée] : http://atilf.atilf.fr/tlf.htm. 
Manning, C. \& Schütze, H. (1999). Foundations of Statistical Natural Language Processing. Cambridge : MIT Press.

Mel'čuk, I., Clas, A. \& Polguère, A. (1995). Introduction à la lexicologie explicative et combinatoire. Louvain-la-Neuve : Duculot.

Mel'čuk, I (dir.) (1984-1999). Dictionnaire explicatif et combinatoire du français contemporain: recherches lexico-sémantiques (4 vol). Montréal: Presses de l'Université.

Mel'čuk, I. (2004a). Actants in semantics and syntax I : actants in semantics. Linguistics, 42(1), pp. 1-66.

Mel'čuk, I. (2004b). Actants in semantics and syntax II : actants in syntax, Linguistics, 42(2), pp. 247-291.

Micheli, R. (2010). L'émotion argumentée. L'abolition de la peine de mort dans le débat parlementaire français. Paris : Cerf.

Moreau, M.-L. (1988). Un mot, des fonctions, des contextes. Linguisticae Investigationes, 12(1), pp. 129-145.

Novakova, I., Grossmann, F. \& Goossens, V. (2013a). Interactions entre profil discursif et structures actancielles : l'exemple des noms de surprise et de respect. In G. Cislaru \& F. Baider (Eds.), Cartographie des émotions. Propositions linguistiques et sociolinguistiques (pp. 71-84). Paris : PSN.

Novakova, I., Grossmann, F. \& Goossens, V. (2013b). Interactions entre profil discursif et structures actancielles: l'exemple des verbes de surprise et de respect. Langue française, 180, pp. 31-46.

Novakova, I. \& Sorba, J. (2013a). Stupéfier et jalouser dans les séquences textuelles journalistiques : quel profil discursif pour quelle stratégie argumentative ?. Le discours et la langue. Revue de linguistique française et d'analyse du discours, 4(1), pp. 203220.

Novakova, I. \& Sorba, J. (2013b). Argumentation et émotion dans les séquences textuelles journalistiques. Le cas de stupeur et de jalousie. In T. Muryn, S. Mejri, W. Prazucc \& I. Sfar (Eds.), La phraséologie entre langues et cultures. Structures, fonctionnements, discours (pp. 137-149). Frankfurt am Main : P. Lang.

Novakova, I. \& Sorba, J. (2014). L'émotion dans le discours. À la recherche du profil discursif de stupeur et de jalousie. In P. Blumenthal, I. Novakova \& D.Siepmann (Eds.), Les émotions dans le discours. Emotions in discourse (pp. 161-173). Frankfurt am Main : P. Lang.

Novakova, I. \& Tutin, A. (Eds.) (2009). Le lexique des émotions. Grenoble : ELLUG.

Plantin, C. (2012). Les séquences discursives émotionnées : définition et application à des données tirées de la base CLAPI. In Actes du CMLF 2012 - $3^{e}$ Congrès Mondial de Linguistique Française (pp. 629-642). Url : http://dx.doi.org/10.1051/shsconf/20120100218.

Rastier, F. (1987). Sémantique interprétative. Paris : PUF.

Rey-Debove, J. \& Rey, A. (Eds.) (2014). Le Petit Robert. Dictionnaire analogique et alphabétique de la langue française. Paris: Dictionnaires Le Robert. Version électronique. 
Tutin, A. (2013). Les collocations lexicales : une relation essentiellement binaire définie par la relation prédicat-argument. Langages, 189, pp. 47-63.

Tutin, A., Novakova, I., Grossmann, F. \& Cavalla, C. (2006). Esquisse de typologie des noms d'affect à partir de leurs propriétés combinatoires. Langue française, 150, pp. 3249.

Van Valin, R. \& LaPolla, R. (1997). Syntax : Structure, Meaning, Function. Cambridge : CUP.

Yannick Mathieu, Y. (2000). Les verbes de sentiments: de l'analyse au traitement automatique. Paris : Éditions du CNRS.

Zhang, L. \& Ferrari. S. (2014). Intensité et polarité : un modèle opératoire articulant plusieurs travaux linguistiques. Langue française, 184, pp. 38-52.

\section{Résumé/Abstracts}

\section{Le rôle du figement dans le traitement de la synonymie au sein du champ de la colère}

Cette étude vise à mettre en évidence, dans une perspective fonctionnelle, les caractéristiques spécifiques de quatre lexies d'affect, présentées comme synonymes, au sein des collocations où elles apparaissent (rage, fureur, rageur, furieux). Les analyses de la combinatoire de ces lexies, menées sur un vaste corpus, permettent d'opérer à trois niveaux (sémantique, syntaxique et textuel) afin d'élargir la description de manière systématique à la dimension discursive. Nous montrons ainsi que le profil discursif de chacune des lexies fournit des éléments pertinents pour distinguer les synonymes entre eux.

Mots-Clés: collocation, phraséologie, figement, synonymie, colère

\section{How the Lexical Fixedness affects the Treatment of Synonymy Among the Semantic Area of Anger}

In this paper, we aim to reveal the distinctive features of the French synonymous lexies rage, fureur, rageur and furieux in the collocations in which each of them appears. Our study is corpus-based. We describe, in the functional perspective, how each of them preferred to be combined with some specific elements in the nearby context and, more generally, in a particular textual environment. Thus, we show that the what we have called 'discursive profile' of each lexie give relevant information to distinguish between these couples of synonyms.

Keywords: collocation, phraseology, lexical restriction, synonymy, anger 
Author's address:

Julie Sorba

Laboratoire LIDILEM

Université Grenoble Alpes

BP 25

F-38040 GRENOBLE Cedex 9

julie.sorba@univ-grenoble-alpes.fr

Vannina Goossens

Laboratoire ICAR

École Normale Supérieure de Lyon

15 parvis René Descartes - BP 7000

F-69342 LYON Cedex 07

vannina.goossens@ens-lyon.fr

\footnotetext{
${ }^{i}$ Nous tenons à remercier chaleureusement Agnès Tutin et Iva Novakova pour leur relecture attentive.

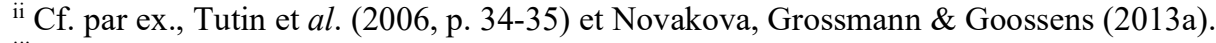

${ }^{i i i}$ Cf. Moreau (1988, p. 144) : « Les utilisateurs du langage ne mémorisent pas les mots comme des unités isolées, mais bien plutôt [...] avec leur contexte ».

iv Comme l'indiquent Van Valin et LaPolla (1997, p. 291) dans le cadre de la Role and Reference Grammar, le choix des arguments syntaxiques n'est pas uniquement prédictible à partir des rôles sémantiques. Il peut être influencé par des facteurs discursifs, en particulier par le statut de topic accordé ou non aux référents des arguments, ce que les auteurs appellent «pragmatic pivot».

${ }^{\mathrm{v}}$ Le terme affect est pris ici comme étiquette conventionnelle permettant de renvoyer aussi bien à des sentiments qu'à des émotions ou à des états affectifs.

${ }^{\text {vi }}$ Cf. Tutin et al. (2006, p. 46).

${ }^{\text {vii }}$ Pour un état des lieux sur la question, cf. Baider \& Constantinou (2014a, p. 56-58) et Baider \& Constantinou (2014b).

viii Dans le Petit Robert, rage est défini comme un « état, mouvement de colère, de dépit extrêmement violent, qui rend agressif » et fureur comme une « colère folle, sans mesure ».

ix Ce corpus est accessible grâce à la plateforme EmoBase (http://emolex.eu/). Le projet EMOLEX (ANR-09-FASHS-017) a permis la constitution d'un vaste corpus annoté morpho-syntaxiquement et syntaxiquement dans cinq langues européennes (français, anglais, allemand, espagnol, russe), dont une partie est alignée.

x L'établissement du lexicogramme d'une lexie pivot sur la base du calcul de log-likelihood (Dunning, 1993 ; Manning \& Schütze, 1999) prend en compte les fréquences de toutes les occurrences du pivot, celles du collocatif et celles de la cooccurrence du pivot et du collocatif. Le lexicogramme ainsi créé
} 
regroupe toutes les cooccurrences présentant une valeur de significativité au moins égale au seuil de spécificité fixé à 10,83. Pour notre étude, nous avons fixé en outre un seuil de fréquence supérieur à 2 .

${ }^{x i}$ La définition de collocation employée dans cette étude combine ainsi un critère statistique de spécificité et des critères sémantico-syntaxiques.

xii La dimension « intensité » a également une valeur 'faible' (légèrement surpris par exemple), pour laquelle nous n'avons trouvé aucun collocatif pour les lexies étud niées ici.

xiii Les pourcentages sont calculés à partir de la somme des valeurs de log-likelihood des collocatifs regroupés par dimension et valeur sémantique.

xiv La dimension "polarité » liée à la valeur axiologique fait référence à des valeurs esthétiques, cognitives, éthiques ou pratiques : « la valeur axiologique des mots ne se réduit pas à leur sens lexical, ils peuvent en acquérir une dans un contexte précis » (Zhang \& Ferrari, 2014, p. 36-37).

${ }^{\mathrm{xv}}$ Tout se passe comme si la polarité très négative présente par défaut dans rage (Baider \& Constantinou, 2014, p. 59) se suffisait à elle-même, sans avoir besoin d'être renforcée par l'expression d'un collocatif. ${ }^{\mathrm{xvi}}$ Contrairement aux collocatifs de manifestation verbale (crier de peur), les collocatifs de verbalisation impliquent un contenu sémantique, la volonté de partager son affect avec un interlocuteur. La valeur « communicative »s'applique à des collocatifs qui dénotent une intention de communication (avouer ses craintes), par opposition à la valeur «émotive» pour laquelle l'échange communicationnel avec l'interlocuteur n'est pas forcément sollicité (crier sa joie).

${ }^{x v i i}$ La transformation de la rage en un autre affect est également possible, mais ces cas sont beaucoup plus rares dans le corpus.

xviii Un actant sémantique d'une lexie L est « une expression qui correspond à un argument du prédicat L [...] cette expression est soit un sens soit une variable dans la définition de L » (Mel'čuk, Clas, Polguère, 1995, p. 76 ; Mel'čuk, 2004a, p. 8). Les actants sémantiques expriment des rôles sémantiques clés comme l'expérienceur, l'objet ou la cause de l'affect, sans correspondre obligatoirement à des actants syntaxiques (Van Valin et LaPolla, 1997).

${ }^{\text {xix }}$ Les exceptions sont au nombre de deux dans notre corpus : sur le ton rageur, $\underline{\grave{a}}$ coups de talon rageur. ${ }^{x x}$ Ce phénomène est également très saillant lorsque l'on compare Les voisins $d u$ Monde et Les voisins d'en face : http://redac.univ-tlse2.fr/applications/vdef.html

${ }^{x x i}$ Nous entendons l'isotopie comme « toute itération d'une unité linguistique » (Rastier, 1987, p. 91) et nous considérons le réseau isotopique comme un procédé qui concoure à la cohésion textuelle.

xxii Ce phénomène saillant se rencontre également avec d'autres lexies d'affect comme stupéfier/stupeur (cf. Novakova \& Sorba, 2013a, 2013b). 